\title{
Disability Prevalence According to a Class, Race, and Sex (CSR) Hypothesis
}

\author{
Carlos Siordia
}

Received: 19 July 2014 /Revised: 13 September 2014 / Accepted: 3 November 2014 /Published online: 6 December 2014

(C) W. Montague Cobb-NMA Health Institute 2014

\begin{abstract}
Disability has been shown to be related in definite ways to social class. In modern industrial societies, disability is influenced by and has the potential to contribute to the production and reproduction of social inequality. However, markers of social stratification processes are sometimes ignored determinants of health. A class, race, sex (CRS) hypothesis is presented to argue that a "low-education disadvantage," "racial-minority disadvantage," and "female disadvantage" will compound to affect the risks for being disable. In particular, the CRS hypothesis posits that class is more important than race and the latter more than sex when predicting presence or severity of disability. The cross-sectional study of community-dwelling adults between the ages of 45 and 64 years uses data from the American Community Survey (ACS) Public Use Microdata Sample (PUMS) 2008-2012 file. By using 3,429,523 individuals - which weighted equal to $61,726,420$ - the results of the study suggest the CRS hypothesis applies to both Non-Latino-Blacks and Non-Latino-Whites. There is a "male disadvantage" exception for NonLatino-Whites. Decreasing between-group differences in health may be achieved by making the age-health association at lower socioeconomic stratum similar to that of the upper socioeconomic strata.
\end{abstract}

Keywords Inequality $\cdot$ Race $\cdot$ Aging $\cdot$ Public health · Function

Electronic supplementary material The online version of this article (doi:10.1007/s40615-014-0073-8) contains supplementary material, which is available to authorized users.

\section{Siordia $(\bowtie)$}

Department of Epidemiology, Graduate School of Public Health, University of Pittsburgh, 130 North Bellefield Ave, Pittsburgh, PA 15213, USA

e-mail: cas271@pitt.edu

\section{Introduction}

This paper merges sociological and epidemiological perspectives to conceptualize how social stratification processes in the USA are associated with disability prevalence as a function of class, race/ethnicity (hereafter only referred to as race), and sex. Disability, defined as having severe limitations with functions of daily living, can be influence by both biological and social factors. The maintenance of homeostasis in physical function can also be affected by place factors - as differently abled individuals (i.e., "the disable") unjustly face social and environmental challenges not encountered by able-bodied people. The current discussion focuses on the social factors associated with disability as work over many decades has consistently shown disability to be related in definite ways to social class. In modern industrial societies, disability is influenced by and has the potential to contribute to the production and reproduction of social stratification.

Authors have previously admonished researchers that "social stratification is essential to understanding and improving health in an aging society" [1]. After explaining that "the greatest potential for increasing the postponement of morbidity and functional health problems lies in making the relation of age to health in lower socioeconomic strata more similar to that in the upper socioeconomic strata," House and colleagues went on to argue that "understanding how this might occur will require that the study of health and aging draw more on basic sociological knowledge of the process of social stratification." This study pays heed to their calls for investigating health as a function of socially stratifying statuses.

The systematic allocation of individuals to different social strata [2] has the ability to influence their ability to obtain (or retain) the resources necessary for maintaining healthy aging. Being relegated to a "low social stratum" is typically accompanied by restricted access to social and economic resources - a phenomenon which may be capable of conferring 
adverse health [3]. Social stratification can harm health by, for example, creating an early onset of frailty and/or increasing the rate of progression toward permanent disability [4]. The systematic relegation to different social strata becomes embodied inequality when it is manifested as between-group health differences (i.e., "health disparities"). Social stratification is an exploitative and oppressive societal phenomenon with health consequences [5]. Many efforts have been undertaken by the US federal government to reduce the influence of social stratification on health. For example, the US congress passed the Minority Health and Health Disparities Research and Education Act of 2000 over a decade ago (Public Law 106-525). The main goal of the public law was to improve minority health by reducing health disparities through a center housed at the National Institutes of Health (NIH). The law states that research on minority health conditions is important as "there has been insufficient research involving such individuals as subjects or insufficient data on such individuals." This study takes advantage of an underutilized data source to provide estimates of disability prevalence for class, race, and sex minorities in the USA.

Work has shown that social status affects health over the life course: where health declines are prevalent in socially disadvantaged by middle age [1]. According to House and colleagues, researchers "should see the largest socioeconomic differentials in health in middle and early old age because these age groups are most likely to be characterized by both sizable socioeconomic differentials in exposure to risk factors and substantial impact of the risk factors" [1]. The primary focus of this paper is to present a conceptual model for how socially stratifying characteristics (e.g., class, race, and sex) are related with disability prevalence in a middle-age range (i.e., ages 45 to 64 years).

Many ecological models have been proposed to explain how, with the advancement of modern medicine, disability would develop in a life span (maximum potential life). For example, Fries' [6] compression of morbidity hypothesis posits that there is a natural limit to life span and a manipulable duration of morbidity before death. Compression of morbidity can occur when the postponement of disease is greater than the postponement of death. In contrast, Gruenberg [7] advanced the idea that life span could be extended by modern medicine. The postponement of death through artificial means, according to Gruenberg's expansion of morbidity hypothesis, could mean that length of time with severe illness before death would increase in the population. Manton [8] advanced the dynamic equilibrium hypothesis, where life span is framed as being affected by medicine and where severe disease before death would also be compressed by advances in medicine.

Neither the compression of morbidity, expansion of morbidity, nor the dynamic equilibrium hypotheses specifically propose how disability would vary over the life span as a function of markers for social stratification. Previous work has shown differences in disability by class $[9,10]$, race $[11,12]$, and sex $[10,13]$. In general, being of lower socioeconomic status, of a racial-minority group, and female is accompanied with greater risk for adverse health. Although many studies have demonstrated between-group differences in health by race, sex, and class, it is less common to find investigations that explore how health varies across their intersections. Analyzing race, sex, and separately may obscured important differences in how disability prevalence is produced and maintained. To help fill this gap in the literature, the current study takes an "intersectionality approach" [14].

A hypothesis on the ecological relationship between social stratum and disability prevalence in mid-life is presented in this study. The hypothesis is informed by empirical findings and predicts that disability is socially stratified first by class, then race, and finally sex. The "class, race, and then sex" (CRS) hypothesis of disability in mid-life for US residents in modern times is visually represented in Fig. 1. The figure shows how disability is predicted to be lowest among those in the highest class - with a "high" level of educational attainment. Within this upper-class group, race is the second stratifying factor - where disability will be lowest among individuals in the race-majority group: Non-Latino-White. Within this majority race group, sex is the third stratifying factorwhere disability, in a patriarchal society, will be lowest in the dominant sex group (i.e., males).

As shown in Fig. 1 and in theory, if class is more important than race and the latter more important than sex, then highly educated males of the majority group would have the lowest disability prevalence from the eight possible group combinations, while the lowest-educated females from the minority group would have the most disability prevalence. The "vitality, to frailty, to disability" morphology is captured in Fig. 1 by the graying zones on the right hand side of the bars. As shown in the figure, the disablement process [15] —according to the ecological CRS hypothesis of disability - would commence at earlier ages among the lowest-educated females from the raceminority group.

More formally, the CRS hypothesis posits that being socially stratified to a low class will burden the individual with a "low-education disadvantage"; to a racial-minority group will penalize the individual with a "racial-minority disadvantage"; and/or to an underpowered sex group will unjustly create a "female disadvantage." The CRS hypothesis in particular posits that class is more important than race and the latter more than sex. As a result, the CRS hypothesis predicts risk for disability will increase with each compounding disadvantage. The specific aim of this study is to use a simple to understand approach with large-scale data to investigate the CRS hypothesis of disability in the US population. The ecological study contributes to the literature by clearly specifying that when it comes to disability prevalence and severity, it will be most clustered by class, then race, then sex. 
Fig. 1 Conceptual representation of disability in middle ages by social stratum

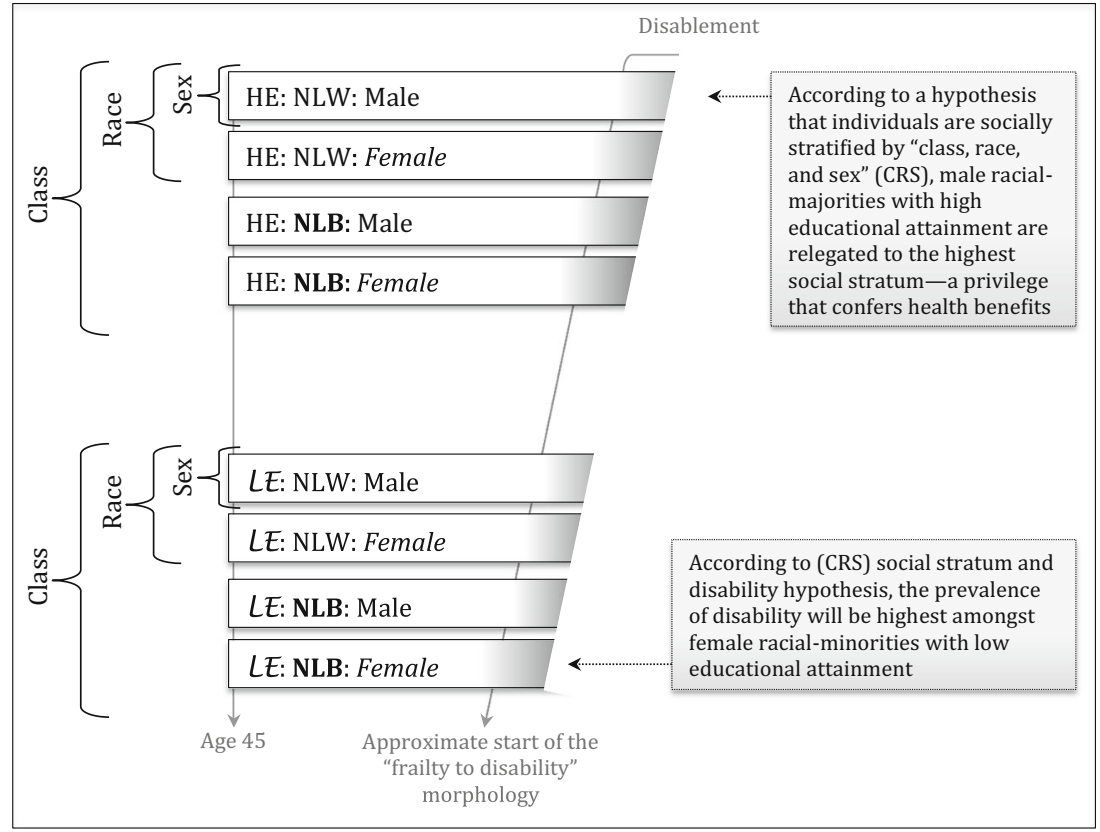

\section{Methods}

\section{Data}

The cross-sectional study uses data from the American Community Survey (ACS). In particular, it uses the Public Use Microdata Sample (PUMS) file from the 5-year survey period of 2008-2012. The US Census Bureau makes the file readily available to anyone with an internet connection. Because the use the de-identified secondary data did not require any person contact, no Internal Review Board approval was required. The project adheres to all ethical guidelines of research. An introductory paper on ACS data has been published [16]. Briefly, the ACS is a cross-sectional yearly survey administered to about $1 \%$ of the population ( $>3$ million people) that uses advance sampling methodologies and collects information through multiple modes [17]. The US federal government uses data from the ACS to influence the allocation of billions of dollars each year. For example, in 2008, ACS data informed the distribution of $\$ 562.2$ billion in grants and $\$ 520.7$ billion in direct payments [18]. There is no other data source on disability in the US population with the same size, transparency, quality, and potential to influence policy.

\section{Sample}

The analytic sample is made up of community-dwelling NonLatino-White (NLW) and Non-Latino-Black (NLB) US citizens, between the ages of ages 45 to 64 years, who only speak English, who have no missing data on variables of interest, and who resided within the contiguous US during the 2008-
2012 survey period. From the 10,151,819 observations in the complete ACS 2008-2012 PUMS 5-year file, the analysis includes a total of 3,429,523 individuals - which weighted equal to $61,726,420$ and of which 10,002,319 (16\%) have at least one disability. Given the survey period and age ranges, individuals in the analysis could have been born between 1944 and 1967. In publications, individuals born within this time frame in the USA are referred to as "baby boomers" [19].

\section{Disability}

Disability is ascertained by determining the presence of one or more difficulties with hearing, seeing, cognition, independent living (e.g., shopping), self-care (e.g., bathing), and ambulation (e.g., walking). The "disability count" variable could thus range from 0 (no difficulties) to 6 (maximum number of difficulties). Details on ACS questions on disability can be readily found on the internet and have been discussed in detail elsewhere [20]. Responses to six survey questions were used to determine if the person has difficulty with any of the items. A person is labeled as being "disabled" if he/she is identified as having difficulties with at least one item. Precision of estimates and measurement issues with disability questions in the ACS have been previously discussed [21, 22].

It is difficult to understand from the estimates of prevalence how "severity" differs between groups. From the total number of disability (ranging from 0 to 6 ) variables used to measure if a person has at least one disability, we see that there are $2,875,756$ (weighted $=51,723,767$ ) with zero disabilities, 285,160 (weighted $=5,135,688$ ) with one disability, 125,415 (weighted $=2,278,847$ ) with two disabilities, 79,433 (weighted $=1,442,981)$ with three disabilities, 45,436 (weighted $=$ 
801,585 ) with four disabilities, 13,796 (weighted $=250,251$ ) with five disabilities, and 4,516 (weighted $=93,301$ ) with six disabilities. These numbers show that about $8 \%$ of the sample has two or more disabilities.

\section{Class and Sex}

Estimates of disability prevalence are stratified by educational attainment, race, and sex. "Class" is measured with a binary variable that labels those with " 1 or more years of college credit, no degree" and beyond (e.g., BA, MS, PhD, and MD) as having a high-education. Those with "some college, but less than 1 year" and below are labeled as having loweducation. Readers should note that class should be measured with more nuance when data allow [23]. The "high" and "low" prefixes on educational attainment level should not be understood as judgment values. These easy-to-follow and simple terms are used to facilitate the grouping of individuals into different social classes. As in other publications [24], the race and ethnicity of the individual are used to identify NLW and NLB individuals. As previously explained in publication [24], the US Census Bureau - the creators of ACS dataconceptualizes race and ethnicity as a social construct and not as a genetically determined and biologically defined phenotype. The population-weighted counts by education, race, sex, and age are presented in Supplementary Table S1.

\section{Statistical Approach}

A SAS ${ }^{\circledR} 9.3$ algorithm (using MACRO) language was created by the author without help from outside sources to produce estimates of sub-population size (i.e., denominators) and estimates on the number of individuals within sub-populations with at least one disability (i.e., numerators). These estimates were used to compute ratios - which are interpreted as percent to discuss prevalence of disability. Please note that the basic epidemiological concept of "prevalence" refers to incidence and duration. While incidence refers to "new cases" (individuals who change from non-disease to disease), duration refers to the average period of time the individual experiences the disease condition. In more epidemiological terms, the current study only accesses "incidence" (i.e., frequency of disability). The term prevalence is used as it may be more readily understood to represent the percent of a population affected by disability. Prevalence in this approach could be argued to include both new and existing cases - it measures the overall number of cases of disability within people surviving in the population.

The SAS ${ }^{\circledR} 9.3$ algorithm computes a total of 320 denominators (shown in Supplementary Table S1) and 320 numerators (shown in Supplementary Table S2) - each of these 640 estimates has a unique standard error which forms the width of the confidence interval (e.g., $95 \%$ Wald upper and lower limits)[25]. More formally, the numerator is the count of individuals in each sub-population who have at least one disability during the survey period. The denominator consists of total number of people within sub-population from which cases of disability arise (i.e., the universe). In addition to the estimation of sub-population disability prevalence, a logistic regression model - predicting the likelihood of having at least one disability (adjusting for age) - is used to investigate how the class-race-sex groups are associated with the risk of being disable. A Poisson regression (adjusting for age) is used to investigate how class-race-sex groups are associated with disability severity.

None of the regression models include a populationweighted variable.

\section{Results}

\section{Disability Prevalence}

Supplementary Table S1 provides the population-weighted total counts by education, race, sex, and age. Supplementary Table S2 provides the population-weighted counts of individuals with at least one disability by education, race, sex, and age. These two tables are combined to produce Fig. 2. For example, there are 714,482 high-education NLW males and 43,851 of them have at least one disability — which means that about $6 \%$ are disabled: $[(43,851 \div 714,482) \times 100]$. A total of 160 ratios, derived by dividing the numbers in Supplementary Table S1 by the corresponding cells in Supplementary Table S2, were produce to create Fig. 2, which clearly shows that disability prevalence is highest among NLBs with loweducation, followed by NLWs with low-education, then NLBs with high-education. As predicted by the CRS hypothesis, disability prevalence is lowest among those with higheducation and from the race-majority group. The overlap in lines by sex challenges the "female disadvantage" posited in the CRS hypothesis - which had predicted that males would have a lower prevalence of disability.

\section{Descriptive Statistics}

Table 1 shows descriptive statistics. The education-race-sex specific weighted and unweighted counts, along with percent disable in the group, are presented in Table 1. The Person Inflation Ratio (PIR) is included as it is deemed an informative value [25]. PIR is the average number of people being represented in weighted population by the unweighted count $=$ (weighted $\div$ unweighted) [25]. As shown in Table 1, on average, high-education NLBs and low-education NLWs represent fewer of their counterparts when population weights are applied. They are followed by high-education NLWs and loweducation NLBs have the largest PIR numbers-indicating 
Fig. 2 Prevalence of disability by race, ethnicity, sex, and educational attainment

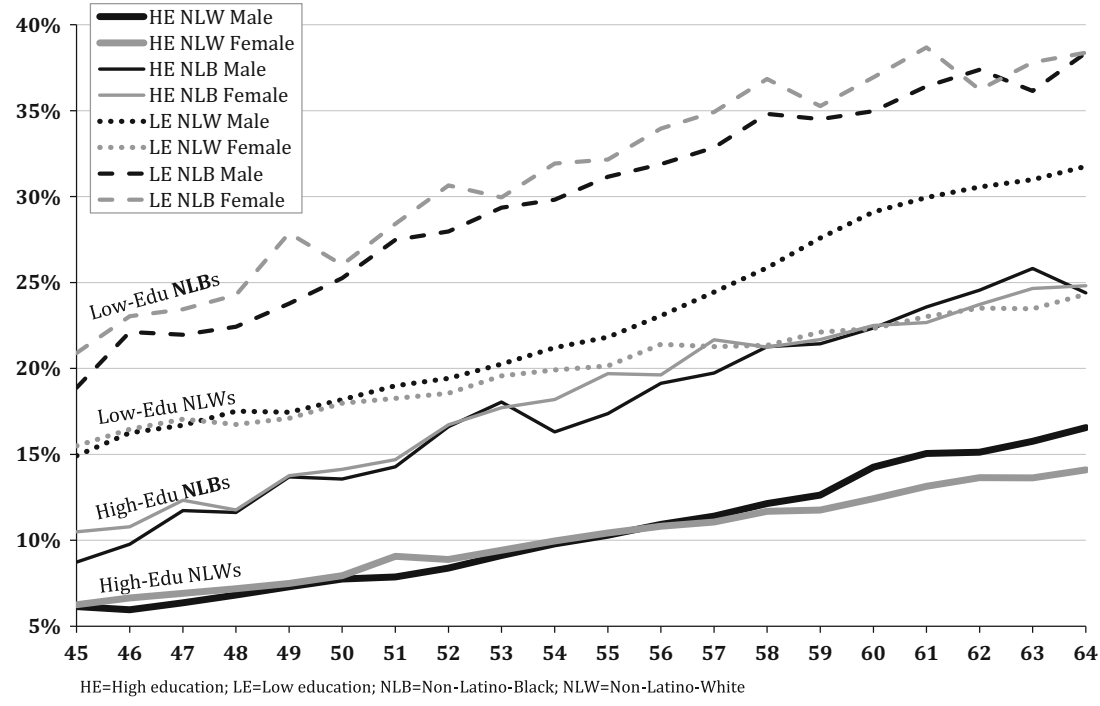

that, on average, they represent more of their counterparts in the population. The pattern in the PIR numbers indicates that population weights in ACS data inflate higheducation NLWs more than low-education NLWs. The PIR pattern reveals an opposite pattern for NLBs, where population weights in ACS data inflate low-education NLBs more than high-education NLBs.

Table 1 also shows that the average age is between 53 and 54 years in all the groups. The table indicates that disability is lowest in high-education NLWs, followed by high-education NLBs, low-education NLWs, and lastly by low-education NLBs. By using the "disability count" variable, the "average severity" for sub-groups is shown in the table. In all the subgroups, the total number of difficulties ranged from the minimum of 0 (non-disable) to the maximum of 6 (most disable). As is made evident by the numbers, average severity follows the CRS hypothesis in all cases except in the NLWs with a low-education where females have lower severity $(0.40)$ relative to males $(0.42)$.

\section{Logistic Regression}

Table 2 shows the results of the logistic model predicting likelihood of having at least one disability. The CRS hypothesis predicted a gradient of risk for disability by class, race, and sex: where risk for disability would be lowest among highly educated NLW males and highest among low-educated NLB females. The regression results partially support the CRS hypothesis. When compared to NLWs males with a high-education, all the other groups, except NLW females with a high-education, have a higher likelihood of being disable after adjusting for age.

The fact that NLW females with a high-education are $5 \%$ less likely to be disabled when compared to NLW males with a high-education contradicts the CRS hypothesis. Similarly, finding that NLW females with a low-education are less likely to be disabled $(112 \%)$ than NLW males with a low-education $(155 \%)$ when compared to NLW males with a high-education

Table 1 Descriptive statistics

\begin{tabular}{|c|c|c|c|c|c|c|c|c|c|}
\hline & Unweighted & Weighted & PIR & Age & SD & Disable & SD & Severity & SD \\
\hline \multicolumn{10}{|l|}{ High-education } \\
\hline NLW, male & 682,431 & $14,519,047$ & 21 & 54 & 24 & 0.10 & 1.29 & 0.17 & 2.61 \\
\hline NLW, female & 706,303 & $15,146,483$ & 21 & 54 & 23 & 0.09 & 1.25 & 0.18 & 2.67 \\
\hline NLB, male & 107,324 & $1,425,525$ & 13 & 53 & 26 & 0.16 & 1.77 & 0.32 & 4.08 \\
\hline NLB, female & 111,362 & $2,080,992$ & 19 & 53 & 25 & 0.17 & 1.75 & 0.33 & 3.99 \\
\hline \multicolumn{10}{|l|}{ Low-education } \\
\hline NLW, male & 804,452 & $11,911,484$ & 15 & 53 & 23 & 0.22 & 1.73 & 0.42 & 4.06 \\
\hline NLW, female & 857,512 & $12,098,572$ & 14 & 54 & 23 & 0.20 & 1.66 & 0.40 & 3.97 \\
\hline NLB, male & 63,339 & $2,270,635$ & 36 & 53 & 25 & 0.28 & 2.08 & 0.58 & 5.17 \\
\hline NLB, female & 96,800 & $2,273,682$ & 23 & 54 & 23 & 0.31 & 2.09 & 0.64 & 5.31 \\
\hline
\end{tabular}

$P I R$ Person Inflation Ratio (weighted $\div$ unweighted), $S D$ standard deviation, $N L B$ Non-Latino-Black, $N L W$ Non-Latino-White 
Table 2 Results of logistic model predicting likelihood of having at least one disability

\begin{tabular}{|c|c|c|c|c|c|}
\hline & OR & LCL & UCL & $\begin{array}{l}\text { Percent } \\
\text { Change }\end{array}$ & \\
\hline \multicolumn{6}{|l|}{ High Education } \\
\hline HE: NLW: Male & 1.00 & ref & ref & ref & \multirow{7}{*}{$\begin{array}{l}\text { NLW-females better than } \\
\text { NLW-males with same } \\
\text { education: } \\
\text { Challenges the CRS hypothesis, } \\
\text { which predicted a "female } \\
\text { disadvantage", by suggesting } \\
\text { a "female advantage" }\end{array}$} \\
\hline HE: NLW: Female & 0.95 & 0.94 & 0.96 & $-5 \%$ & \\
\hline HE: NLB: Male & 1.96 & 1.92 & 2.00 & $96 \%$ & \\
\hline HE: NLB: Female & 2.00 & 1.97 & 2.04 & $100 \%$ & \\
\hline Low Education & & & & & \\
\hline LE: NLW: Male & 2.55 & 2.53 & 2.57 & $155 \%$ & \\
\hline LE: NLW: Female & 2.12 & 2.10 & 2.14 & $112 \%$ & \\
\hline LE: NLB: Male & 4.05 & 3.99 & 4.11 & $305 \%$ & \\
\hline LE: NLB: Female & 4.33 & 4.27 & 4.39 & $333 \%$ & \\
\hline Age & 1.05 & 1.04 & 1.05 & $5 \%$ & \\
\hline
\end{tabular}

All $\beta$ values are statistically significant at an $\alpha$ level $<0.001$. Percent change $=[(\mathrm{OR}-1) \times 100]$

OR odds ratio, $L C L 95 \%$ Wald lower confidence limit, UCL $95 \%$ Wald upper confidence limit, HE high education, $L E$ low-education, $N L B$ Non-LatinoBlack, NLW Non-Latino-White

also contradicts the CRS hypothesis. As highlighted in Table 2, the female disadvantage predicted by the CRS hypothesis is absent within NLWs.

Thus, the regression results suggest that the CRS hypothesis fully applies to NLBs and necessitates an exception for NLWs. For NLBs, the risk for disability does compound by class, race, and sex as predicted by the CRS hypothesis. For NLWs, risk for disability compounds by class, race, and sex-but unlike the CRS hypothesis positing a female disadvantage, among NLWs, there is a "male disadvantage." Despite the nuance on the sex factor for NLWs, for both NLWs and NLBs - as predicted by the CRS hypothesis - class is more important than race and the latter more than sex.

\section{Poisson Regression}

Table 3 shows the results of the Poisson model predicting disability severity. The results lend further support for the CRS hypothesis. There is almost a clear gradient in how the markers of social stratification relate with disability severity. The results indicate that there are low-education, minorityrace, and female disadvantage when it comes to disability severity in all groups except low-education NLWs where the "male disadvantage" is again made present. As predicted by the CRS hypothesis, when compared to high-education NLW males, the other groups follow a clear gradient of disability risk from least to most as follows: high-education NLW females $(\beta=0.01)$, high-education NLB males $(\beta=0.70)$, high-education NLB females $(\beta=0.71)$, low-education NLW females $(\beta=0.81)$, low-education NLW males $(\beta=0.92)$, loweducation NLB males $(\beta=1.32)$, and low-education NLB females $(\beta=1.38)$.

The CRS hypothesis is fully supported when modeling disability severity in this sample. For NLBs, disability severity compounds by class, race, and sex as predicted by the CRS hypothesis. For NLWs, disability severity compounds by class, race, and sex - with the exception of a male disadvantage among low-education NLWs. As with the interpretations of the results from the logistic model, the Poisson regression outputs show that for both NLWs and NLBs - as predicted by the CRS hypothesis - class is more important than race and the latter more than sex.

\section{Conclusions}

The study finds support the CSR hypothesis. The results of the study suggest that for NLBs, there is low-education, racialminority, and female disadvantage when predicting either presence or severity of disability. The results of the study suggest that for NLWs, there is low-education, racial-minority, 
Table 3 Results of Poisson model predicting severity of disability

\begin{tabular}{|c|c|c|c|c|}
\hline & $\beta$ & LCL & UCL & \\
\hline \multicolumn{5}{|l|}{ High Education } \\
\hline NLW: Male & 1.00 & ref & ref & \\
\hline NLW: Female & 0.01 & 0.01 & 0.02 & \\
\hline NLB: Male & 0.70 & 0.69 & 0.72 & \\
\hline NLB: Female & 0.71 & 0.70 & 0.73 & \multirow{6}{*}{$\begin{array}{l}\text { NLW-females better than } \\
\text { NLW-males with same } \\
\text { education: } \\
\text { Challenges the CRS hypothesis, } \\
\text { which predicted a "female } \\
\text { disadvantage", by suggesting a } \\
\text { "female advantage" }\end{array}$} \\
\hline Low Education & & & & \\
\hline NLW: Male & 0.92 & 0.92 & 0.93 & \\
\hline NLW: Female & 0.81 & 0.81 & 0.82 & \\
\hline NLB: Male & 1.32 & 1.31 & 1.33 & \\
\hline NLB: Female & 1.38 & 1.38 & 1.39 & \\
\hline Age & 0.04 & 0.03 & 0.04 & \\
\hline
\end{tabular}

All $\beta$ values are statistically significant at an $\alpha$ level $<0.001$

$L C L 95 \%$ Wald lower confidence limit, $U C L 95 \%$ Wald upper confidence limit, NLB Non-Latino-Black, NLW Non-Latino-White

and male disadvantage when predicting either presence or severity of disability. Descriptive statistics and regression results support the CRS hypothesis predicting that class would be more important than race and that the latter would be more important than sex. The general finding may be interpreted as indicating that social stratification, when embodied as presence or severity of disability, functions first by class, then race, and finally by sex.

There are limitations with the current study. Foremost is the fact that the cross-sectional approach prohibits us from understanding if the disadvantaged statuses produced disability in middle age or if disability itself begat the low-education status. That is, the presence of differences in disability prevalence by educational attainment at mid-life may reflect the impact of health on class rather than vice versa. The relationship between social stratification and disability is complex and may be bidirectional as disablement has the ability to initiate or aggravate a disadvantaged [26] and being stratified to low stratum may precede disability [27]. Cross-sectionally, a direct relationship between disability and SES is frequently found [28] and evidence abounds on the gradient of disability by socioeconomic status. Although a causal relationship between social and economic disadvantage and disability is still being debated, it may be plausible that being relegated to lower social stratum more frequently affects the commencement and advancement of the disablement process than vice versa [29]. Future work should continue to inves- tigate if low socioeconomic status in early life more frequently precedes disability in mid-life. The regression models are also limited in that they do not measure comorbidity. Researchers should investigate this topic with data that includes measures of comorbidity. The study only uses an arbitrary educational attainment level to measure class. Publications do show that during this time period, about one in every four individuals in the USA had a college education [30]. Future work should explore more nuanced measures of class and alternate thresholds in educational attainment.

Notwithstanding limitations, the study is the first to show that when modeling presence of severity of disability, social stratification plays a role by compounding penalties first by class, then by race, and finally by sex. The study supports existing research finding disability to be related in definite ways to social class $[31,32]$. Understanding how things like cellular senescence produce degenerative diseases that affect organs to compromise physiological capacity necessitates that markers of social stratification be considered. Genes, biology, and the environment affect health. The propensity for disability is partly dependent on their interaction. The development of effective interventions necessitates that markers of social stratification be included when outlining the non-random mechanisms by which betweengroup differences are created. The study introduces and supports the CSR hypothesis by using information on 
more than 3.4 million people in the USA. Public health researchers and epidemiologist should continue to treat markers of social stratification processes as important determinants of health.

Ethical Standard Statement The author does not have a financial relationship with the organization that sponsored the research. Carlos Siordia is supported by the National Institute of Aging at the National Institutes of Health (grant number T32 AG000181 to A. B. Newman). The author declares that he has no conflict of interest.

Informed Consent The investigation did not require contact with human subjects. No IRB approval was obtained as only secondary, publicly available, and de-identified data was used.

\section{References}

1. House JS et al. The social stratification of aging and health. Journal of Health and Social Behavior. 1994;213-234

2. Feagin J. Systemic racism: a theory of oppression. Routledge. 2013.

3. Ndiaye $\mathrm{K}$ et al. Health disparities and discrimination: three perspectives. J Health Disparities Res Pract. 2012;2(3):4.

4. Liang J et al. Social stratification and trajectories of functional status among older Taiwanese. 健康與社會. 2013;1(1):65-94.

5. Krieger N. Methods for the scientific study of discrimination and health: an ecosocial approach. Am J Public Health. 2012;102(5): 936-44.

6. Fries JF. Aging, natural death, and the compression of morbidity. N Engl J Med. 1980;303:130-5.

7. Gruenberg EM. The failures of success. Milbank Q. 2005;83:779800.

8. Manton KG. Changing concepts of morbidity and mortality in the elderly population. Milbank Memorial Fund Q, Health Soc. 1982;60: 183-244.

9. Minkler M, Fuller-Thomson E, Guralnik JM. Gradient of disability across the socioeconomic spectrum in the United States. N Engl J Med. 2006;355(7):695-703.

10. Melzer D et al. Socioeconomic status and the expectation of disability in old age: estimates for England. J Epidemiol Community Health. 2000;54(4):286-92.

11. McDonald KE, Keys CB, Balcazar FE. Disability, race/ethnicity and gender: themes of cultural oppression, acts of individual resistance. Am J Community Psychol. 2007;39(1-2):145-61.

12. Warner DF, Brown TH. Understanding how race/ethnicity and gender define age-trajectories of disability: an intersectionality approach. Soc Sci Med. 2011;72(8):1236-48.

13. Leveille SG et al. Sex differences in the prevalence of mobility disability in old age: the dynamics of incidence, recovery, and mortality. J Gerontol Series B. 2000;55(1):S41-50.

14. Mullings L, Schulz AJ. Intersectionality and health: an introduction. Jossey-Bass. 2006.
15. Schulz R, Heckhausen J, O'Brien A. The disablement process in late life: a life-span control theory approach. In: Manuck, Jennings, Rabin, Baum, editors. Behavior, health, and aging. New York: Taylor \& Francis Group; 2014. p. 119-32.

16. Herman E. The American Community Survey: an introduction to the basics. Gov Inf Q. 2008;25(3):504-19.

17. Siordia C. Detecting "real" population changes with American Community Survey data: the implicit assumption of treating between-year differences as "trends.". J Sociol Res. 2014;4(2):494509.

18. Reamer AD. Surveying for dollars: the role of the American Community Survey in the geographic distribution of federal funds. Metropolitan Policy Program at Brookings, Washington D.C. 2010.

19. Siordia C, Leyser-Whalen O. The lucky few and baby boom generation: relative cohort size, Mexican Ethnicity, and gender on the likelihood of out-of-poverty status. Sociol Focus. 2014;47(3):16373.

20. Siordia C, Young RL. Methodological note: allocation of disability items in the American Community Survey. Disabil Health J. 2013;6(2):149-53.

21. Siordia C. Precision in estimates of disability prevalence for the population aged 65 and over in the United States by race and ethnicity. Journal of Frailty and Aging. 2014; ahead of print: http:// www.jfrailtyaging.com/all-issues.html?article $=193$.

22. Siordia C. Proxy-reports in the Ascertainment of Disability Prevalence with American Community Survey Data. Journal Frailty and Aging. 2014; ahead of print: http://www.jfrailtyaging. com/all-issues.html?article $=188$.

23. Mason K. Social stratification and the body: gender, race, and class. Sociol Compass. 2013;7(8):686-98.

24. Siordia C. Number of people in the Unites States experiencing ambulatory and independent living difficulties. Journal of Social Work and Disability and Rehabilitation. 2013;13(3.1).

25. Siordia C, Le VD. Precision of disability estimates for southeast Asians in American Community Survey 2008-2010 microdata. Central Asian J Global Health. 2013;1(2):doi:10.5195/cajgh.2012. 2166-7403.

26. Trani JF, Loeb M. Poverty and disability: a vicious circle? Evidence from Afghanistan and Zambia. J Int Dev. 2012;24:S19-52.

27. Lustig DC, Strauser DR. Causal relationships between poverty and disability. Rehabil Couns Bull. 2007;50:194-202.

28. Pandey MK. Poverty and disability among Indian elderly evidence from household survey. J Disabil Policy Stud. 2012;23: $39-49$.

29. Adler NE, Ostrove JM. Socioeconomic status and health: what we know and what we don't. Ann N Y Acad Sci. 1999;896:3-15.

30. Ryan CL, Siebens J. Educational attainment in the United States: 2009. American Community Survey Reports, US Department of Commerce. 2012, P20-566.

31. Jenkins R. Disability and social stratification. British Journal of Sociology. 1991;557-580.

32. Woolf SH, Aron LY. The US health disadvantage relative to other high-income countries: findings from a National Research Council/Institute of Medicine report. JAMA. 2013;309(8): $771-2$. 